\title{
TITLE:
}

\section{Three-quark potential and Abelian dominance of confinement in SU(3) QCD}

AUTHOR(S):

Sakumichi, Naoyuki; Suganuma, Hideo

\section{CITATION:}

Sakumichi, Naoyuki ...[et al]. Three-quark potential and Abelian

dominance of confinement in SU(3) QCD. Physical Review D 2015, 92(3): 034511.

ISSUE DATE:

2015-08-21

URL:

http://hdl.handle.net/2433/199648

RIGHT:

(C) 2015 American Physical Society. 
PHYSICAL REVIEW D 92, 034511 (2015)

\title{
Three-quark potential and Abelian dominance of confinement in SU(3) QCD
}

\author{
Naoyuki Sakumichi ${ }^{1}$ and Hideo Suganuma ${ }^{2}$ \\ ${ }^{1}$ Theoretical Research Division, Nishina Center, RIKEN, Wako, Saitama 351-0198, Japan \\ ${ }^{2}$ Department of Physics, Kyoto University, Kitashirakawaoiwake, Sakyo, Kyoto 606-8502, Japan \\ (Received 1 February 2015; published 21 August 2015)
}

\begin{abstract}
We study the baryonic three-quark (3Q) potential and its Abelian projection in terms of the dualsuperconductor picture in SU(3) quenched lattice QCD. The non-Abelian SU(3) gauge theory is projected onto Abelian $\mathrm{U}(1)^{2}$ gauge theory in the maximal Abelian gauge. We investigate the $3 \mathrm{Q}$ potential and its Abelian part for more than 300 different patterns of static $3 \mathrm{Q}$ systems in total at $\beta=5.8$ on $16^{3} 32$ and at $\beta=6.0$ on $20^{3} 32$ with $1000-2000$ gauge configurations. For all the distances, both the $3 Q$ potential and Abelian part are found to be well described by the Y ansatz, i.e., two-body Coulomb term plus three-body Y-type linear term $\sigma_{3 \mathrm{Q}} L_{\min }$, where $L_{\min }$ is the minimum flux-tube length connecting the three quarks. We find equivalence between the three-body string tension $\sigma_{3 \mathrm{Q}}$ and its Abelian part $\sigma_{3 \mathrm{Q}}^{\text {Abel }}$ with an accuracy within a few percent deviation, i.e., $\sigma_{3 Q} \simeq \sigma_{3 Q}^{\text {Abel }}$, which means Abelian dominance of the quark-confining force in $3 \mathrm{Q}$ systems.
\end{abstract}

DOI: 10.1103/PhysRevD.92.034511

PACS numbers: 11.15.Ha, 12.38.Aw, 12.38.Gc

\section{INTRODUCTION}

Quark confinement has been one of the most important long-standing issues remaining in theoretical physics [1] since the concept of quarks was introduced in the 1960s. In fact, quarks cannot be observed individually and are confined in color-singlet combinations of mesons or baryons. In particular, the nucleon, the lightest baryon, is one of the main ingredients of the matter in our real world, and, therefore, the quark confinement in baryons or three-quark (3Q) systems would be fairly important in modern physics, as well as in mesons or quark-antiquark (QQ̄) systems. Furthermore, the three-body force among three quarks is a "primary" force reflecting the $\mathrm{SU}(3)$ gauge symmetry in quantum chromodynamics (QCD) [2,3], while the three-body force appears as a residual interaction in most fields of physics. Nevertheless, the quark interaction in baryonic 3Q systems [2-4] has not been investigated so much, in contrast with many lattice studies on QQ $\bar{Q}$ systems $[1,5,6]$.

In SU(3) quenched lattice QCD, the static QQ $[5]$ and 3Q $[2,3,7]$ potentials are found to be well reproduced by

$$
\begin{gathered}
V(r)=\sigma r-\frac{A}{r}+C \\
V_{3 \mathrm{Q}}\left(\mathbf{r}_{1}, \mathbf{r}_{2}, \mathbf{r}_{3}\right)=\sigma_{3 \mathrm{Q}} L_{\min }-\sum_{i<j} \frac{A_{3 \mathrm{Q}}}{\left|\mathbf{r}_{i}-\mathbf{r}_{j}\right|}+C_{3 \mathrm{Q}},
\end{gathered}
$$

respectively. Here, $\mathbf{r}_{1}, \mathbf{r}_{2}$, and $\mathbf{r}_{3}$ are the positions of the three quarks, and $L_{\min }$ is the minimum flux-tube length connecting the three quarks as shown in Fig. 1(a). The form (2) is called the Y ansatz [3]. These functional forms (1) and (2) indicate the flux-tube picture [8] on the confinement mechanism. In fact, the lattice QCD simulations [1,9-13] on the action density in the presence of a static QQ system have actually shown the flux-tube formation; that is, valence quarks are linked by the color flux tube as a quasione-dimensional object. Here, the strength of quark confinement is controlled by the string tension of the flux tube, $\sigma$ or $\sigma_{3 \mathrm{Q}}$. We also note that the baryonic 3Q system has recently received attention in the context of the holographic description of strong interactions, e.g., AdS/QCD effective string theories [14].

The difficulty in deriving quark confinement directly from QCD is considered to originate from non-Abelian dynamics and nonperturbative features of QCD, which are quite different from the case of quantum electrodynamics (QED). However, it remains unclear whether quark confinement is peculiar to the non-Abelian nature of QCD or not.
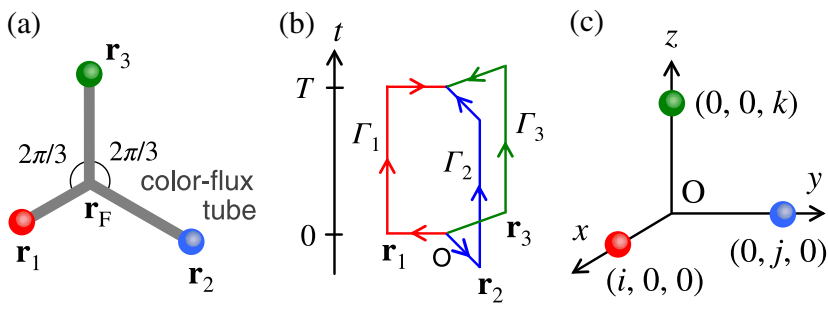

FIG. 1 (color online). (a) The flux-tube configuration of the three-quark system with the minimal value of the total flux-tube length. There appears a physical junction linking the three flux tubes at the Fermat point $\mathbf{r}_{F}$. (b) The trajectory of the 3Q Wilson loop $W_{3 \mathrm{Q}}$. The three quarks are generated at $t=0$, are spatially fixed in $\mathbb{R}^{3}$ for $0<t<T$, and are annihilated at $t=T$. (c) The configuration of static three-quark sources in our lattice QCD simulations. 
NAOYUKI SAKUMICHI AND HIDEO SUGANUMA

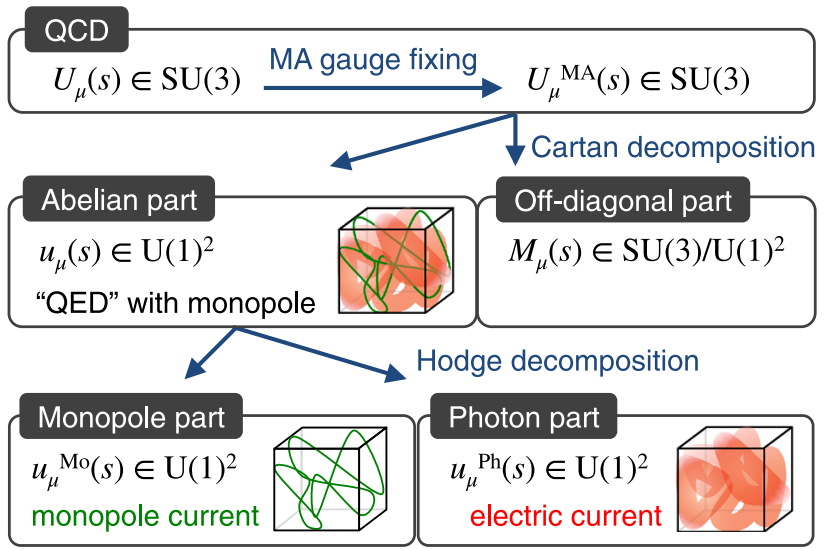

FIG. 2 (color online). Schematic figure of Abelianization of QCD and the dual-superconductor scenario of confinement. In the MA gauge, QCD becomes Abelian-like, and the monopole current topologically appears. By the Hodge decomposition, the QCD system can be divided into the monopole and the photon parts. The monopole part has confinement, chiral symmetry breaking, and instantons, while the photon part does not have all of them [21,23-25].

As an interesting idea of quark confinement, Nambu, 't Hooft, and Mandelstam proposed an Abelian theory of the dual superconductor for the confinement mechanism [15] in the 1970s. In the dual-superconductor picture, the squeezing of the color-electric flux among quarks is realized by the dual Meissner effect as the result of condensation of color-magnetic monopoles. (Note here that monopole condensation and its relevant role for confinement have been analytically pointed out by Seiberg and Witten in the $N=2$ supersymmetric version of the Yang-Mills theory [16].)

As for the possible connection between the dual superconductor and QCD, 't Hooft proposed a concept of "Abelian projection" as an infrared Abelianization scheme of QCD [17,18], where the magnetic monopole topologically appears. And 't Hooft also conjectured that longdistance physics such as confinement could be realized only by Abelian degrees of freedom in QCD [17], which is called "(infrared) Abelian dominance." Actually, in the maximally Abelian (MA) gauge [19-22], QCD becomes Abelian-like as a result of a large off-diagonal gluon mass of about $1 \mathrm{GeV}$ [22], and the monopole current topologically appears [19]. (See Fig. 2.) By using the Hodge decomposition, the QCD vacuum can be divided into the monopole and the photon parts. The lattice QCD studies demonstrate that the monopole part has confinement [21], chiral symmetry breaking [23,24], and instantons [25], while the photon part does not have all of them.

Many lattice QCD studies have remarkably shown Abelian dominance of the confining force in static QQ systems in the MA gauge: the string tension $\sigma$ is reproduced by the Abelian-projected one $\sigma^{\text {Abel }}$ in both SU(2) [19-22]
PHYSICAL REVIEW D 92, 034511 (2015)

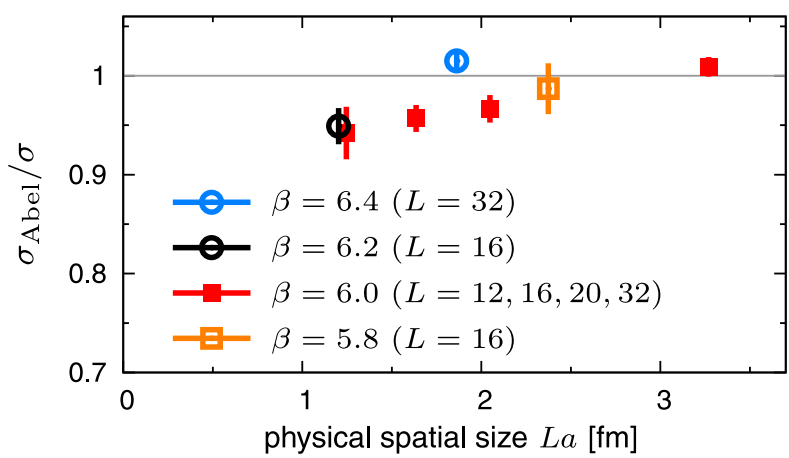

FIG. 3 (color online). Physical spatial-size dependence of $\sigma_{\text {Abel }} / \sigma$ taken from Ref. [28]. Here, $\sigma$ and $\sigma_{\text {Abel }}$ are the string tensions of the QQ potential for SU(3) QCD and the Abelian part, respectively. Perfect Abelian dominance $\left(\sigma_{\mathrm{Abel}} / \sigma \simeq 1\right)$ seems to be realized when the spatial size $L a$ is larger than about $2 \mathrm{fm}$. In this paper, we investigate the corresponding string tensions of $3 \mathrm{Q}$ potentials for $\beta=5.8$ on $16^{3} 32$ and $\beta=6.0$ on $20^{3} 32$ lattices.

and SU(3) [26,27] color QCD. Recently, in the SU(3) quenched lattice QCD, we found perfect Abelian dominance [28] of the quark-confining force in QQ $\bar{Q}$ systems; i.e., the confining force is entirely reproduced only with the Abelian sector, $\sigma^{\text {Abel }} \simeq \sigma$. (To be precise, e.g., $\sigma^{\text {Abel }} / \sigma=$ 1.01(1) and 1.00(2) for $\beta=6.0$ on $32^{4}$ lattice and $\beta=5.8$ on $16^{3} 32$ lattice, respectively.) To obtain $\sigma^{\text {Abel }} \simeq \sigma$, it is necessary to use (i) a larger numbers of gauge configurations, (ii) both on-axis and several types of off-axis data, and (iii) large-volume lattices of more than about $2 \mathrm{fm}$. In particular, the use of large physical-volume lattices is essential, as is shown in Fig. 3 [28]. Moreover, very recently, perfect Abelian dominance was reported also in SU(2) quenched QCD [29]. [The authors of Ref. [29] reported $\sigma^{\text {Abel }} / \sigma=1.02(2)$ for $\beta=2.5$ on $24^{4}$ lattice, where the physical volume is $L a \simeq 2.0 \mathrm{fm}$.] These observations of $\sigma^{\text {Abel }} \simeq \sigma$ indicate that the Abelianization of QCD can be realized without loss of the quark-confining force via the MA projection.

In this paper, we investigate whether quark confinement in the baryonic 3Q potential is entirely kept in the Abelian sector of QCD in the MA gauge and find this to be true at the quenched level. Despite the physical importance of baryons, there are very little studies about Abelian dominance in the baryonic $3 Q$ potential [10] because the previous lattice studies of Abelian dominance were performed mainly for simplified SU(2) color QCD, where the color structure of SU(2) baryons (QQ) are the same as that of mesons (QQ̄). In a pioneering study, Bornyakov et al. [10] reported approximate Abelian dominance of the string tension in the 3Q potential, $\sigma_{3 \mathrm{Q}}^{\mathrm{Abel}} / \sigma_{3 \mathrm{Q}}=0.83(3)$, using the simulated annealing algorithm to avoid Gribov copy effects, on a $16^{3} 32$ lattice at $\beta=6.0$. However, from the results of $\sigma^{\mathrm{Abel}} \simeq \sigma$ in mesonic QQ̄ cases [28], it is expected that the equivalence $\sigma_{3 \mathrm{Q}}^{\mathrm{Abel}} \simeq \sigma_{3 \mathrm{Q}}$ can be also realized in 
baryonic $3 \mathrm{Q}$ cases. To investigate the equivalence $\sigma_{3 \mathrm{Q}}^{\text {Abel }} \simeq \sigma_{3 \mathrm{Q}}$, it seems necessary to use (i) large numbers of gauge configurations, (ii) larger numbers of $3 \mathrm{Q}$ configurations, and (iii) large-volume lattices of more than about $2 \mathrm{fm}$, which are inspired from the analysis on the quark-confining force in mesons [28]. Therefore, in this paper, we perform the accurate calculation that meets the above conditions. Then, we find equivalence $\sigma_{3 \mathrm{Q}}^{\text {Abel }} \simeq \sigma_{3 \mathrm{Q}}$ within a few percent deviation.

\section{NUMERICAL SETTING FOR MAXIMAL ABELIAN PROJECTION}

We perform the SU(3) quenched lattice QCD simulations with the standard plaquette action. We mainly use the lattice of $L^{3} L_{t}=16^{3} 32$ at $\beta \equiv 6 / g^{2}=5.8$, with the gauge coupling $g$, the spatial size $L^{3}$, and the temporal one $L_{t}$. The lattice spacing is $a=0.148(2) \mathrm{fm}$, which is determined so as to reproduce the string tension $\sigma=0.89 \mathrm{GeV} / \mathrm{fm}$ in the $\mathrm{QQ}$ potential. Thus, the physical spatial volume of the lattice is estimated as $(2.37(3) \mathrm{fm})^{3}$. We also use a finer lattice of $20^{3} 32$ at $\beta=6.0$, which corresponds to $a=$ $0.1022(5) \mathrm{fm}$ and the physical spatial volume of $(2.05(1) \mathrm{fm})^{3}$. The simulation conditions are summarized in Table I.

For $\beta=5.8$ and 6.0 , we use 2000 and 1000 gauge configurations, respectively, which are taken every 500 sweeps after a thermalization of 20000 sweeps. It is worth mentioning that the used configuration number 2000 is about ten times larger than that in the previous detailed lattice studies of baryonic 3Q potentials [2,3]. The large number of the gauge configurations enables us to measure accurately the large-distance $3 \mathrm{Q}$ potential data, which is important for the confinement study.

In the lattice formalism, the $\mathrm{SU}(3)$ gauge field is described by the link variable $U_{\mu}(s)=e^{i a g A_{\mu}(s)} \in \mathrm{SU}(3)$ instead of the gluon field $A_{\mu}(s) \in \operatorname{su}(3)$. We perform the SU(3) MA gauge fixing by maximizing

$$
\begin{aligned}
R_{\mathrm{MA}}\left[U_{\mu}(s)\right] & \equiv \sum_{s} \sum_{\mu=1}^{4} \operatorname{tr}\left(U_{\mu}^{\dagger}(s) \vec{H} U_{\mu}(s) \vec{H}\right) \\
& =\frac{1}{2} \sum_{s} \sum_{\mu=1}^{4}\left(\sum_{i=1}^{3}\left|U_{\mu}(s)_{i i}\right|^{2}-1\right)
\end{aligned}
$$

under the $\mathrm{SU}(3)$ gauge transformation $U_{\mu}(s) \mapsto$ $\Omega(s) U_{\mu}(s) \Omega^{\dagger}(s+\hat{\mu})$ with $\Omega(s) \in \mathrm{SU}(3)$. Here, $\vec{H}=$ $\left(T_{3}, T_{8}\right)$ is the Cartan subalgebra of $\mathrm{SU}(3)$, and $T_{3}=$ $\operatorname{diag}(1 / 2,-1 / 2,0)$ and $T_{8}=(1 / 2 \sqrt{3}) \times \operatorname{diag}(1,1,-2)$ in the standard notation. (The functional (3) has been used for MA gauge fixing in Refs. [10,26-28,30,31].) We numerically maximize $R_{\mathrm{MA}}$ for each gauge configuration $\left\{U_{\mu}(s)\right\}$ until it converges, by using the over-relaxation method $[26,28]$. As for the stopping criterion, we stop the
TABLE I. The simulation condition: $\beta$, the lattice size $L^{3} L_{t}$, and the gauge-configuration number $N_{\text {con }}$. The corresponding lattice spacing $a$ and the physical spatial size $L a$ are also listed. Here, the values in parentheses denote the standard error.

\begin{tabular}{lllll}
\hline \hline$\beta$ & $L^{3} L_{t}$ & $N_{\text {con }}$ & $a(\mathrm{fm})$ & $L a(\mathrm{fm})$ \\
\hline 5.8 & $16^{3} 32$ & 2000 & $0.148(2)$ & $2.37(3)$ \\
6.0 & $20^{3} 32$ & 1000 & $0.1022(5)$ & $2.05(1)$ \\
\hline \hline
\end{tabular}

maximization algorithm, when the deviation $\Delta R_{\mathrm{MA}} /\left(4 L^{3} L_{t}\right)<10^{-9}$ after the one-sweep gauge transformation. From Eq. (3), we remark $-1 / 2 \leq$ $R_{\mathrm{MA}} /\left(4 L^{3} L_{t}\right) \leq 1$ for arbitrary gauge configuration $\left\{U_{\mu}(s)\right\}$. The converged value of $\left\langle R_{\mathrm{MA}}\right\rangle /\left(4 L^{3} L_{t}\right)$ is $0.7072(6)$ at $\beta=5.8$ and $0.7322(5)$ at $\beta=6.0$, where $\langle\cdots\rangle$ is the statistical average over the gauge configurations and the value in parentheses denotes the standard deviation. Note that the maximized value of $R_{\mathrm{MA}}$ is almost the same over 1000-2000 gauge configurations because the standard deviation of $R_{\mathrm{MA}}$ is fairly small. Then, we expect that our procedure escapes bad local minima, where $R_{\mathrm{MA}}$ is relatively small, and the Gribov copy effect is not significant.

We extract the Abelian part of the link variable,

$$
u_{\mu}(s)=\exp \left(i \theta_{\mu}^{3}(s) T_{3}+i \theta_{\mu}^{8}(s) T_{8}\right) \in \mathrm{U}(1)_{3} \times \mathrm{U}(1)_{8},
$$

by maximizing the norm

$$
R_{\text {Abel }} \equiv \frac{1}{3} \operatorname{Retr}\left(U_{\mu}^{\mathrm{MA}}(s) u_{\mu}^{\dagger}(s)\right) \in\left[-\frac{1}{2}, 1\right],
$$

where $U_{\mu}^{\mathrm{MA}}(s) \in \mathrm{SU}(3)$ denotes the link variable in the MA gauge. In the MA gauge, there remains the residual $\mathrm{U}(1)^{2}$ gauge symmetry with the global Weyl (color permutation) symmetry [32]. In fact, $R_{\mathrm{MA}}$ in Eq. (3) is invariant under the $\mathrm{U}(1)^{2}$ gauge transformation $U_{\mu}(s) \mapsto$ $\omega(s) U_{\mu}(s) \omega^{\dagger}(s+\hat{\mu})$ with $\omega(s) \in \mathrm{U}(1)_{3} \times \mathrm{U}(1)_{8}$ and the global color permutation. Under the $\mathrm{U}(1)^{2}$ gauge transformation, the Abelian link variable $u_{\mu}(s)$ transforms as

$$
u_{\mu}(s) \mapsto \omega(s) u_{\mu}(s) \omega^{\dagger}(s+\hat{\mu}),
$$

which means that $u_{\mu}(s)$ behaves as a $\mathrm{U}(1)^{2}$ gauge field. Here, the MA-projected $\mathrm{U}(1)^{2}$ Abelian theory is similar to compact QED, and it has not only the electric current but also the magnetic-monopole current.

Since off-diagonal-gluon components are suppressed in the MA gauge, we find approximate "microscopic Abelian dominance" [32] for the Abelian link variable as $u_{\mu}(s) \simeq$ $U_{\mu}^{\mathrm{MA}}(s)$ or $\left\langle R_{\text {Abel }}\right\rangle \simeq 1$, i.e., $\left\langle R_{\text {Abel }}\right\rangle=0.8924(3)$ at $\beta=$ 5.8 and $0.9027(2)$ at $\beta=6.0$. However, it is a highly nontrivial question whether this gauge shows "macroscopic Abelian dominance" such as Abelian dominance of quark confinement in QQ $\bar{Q}$ and $3 \mathrm{Q}$ potentials. 


\section{NUMERICAL CALCULATION METHOD FOR THREE-QUARK POTENTIAL}

Similar to the case of the QQ potential $V(r)$ [28], the color-singlet baryonic $3 \mathrm{Q}$ potential $V_{3 \mathrm{Q}}$ can be calculated as $[2,3,33]$

$$
V_{3 \mathrm{Q}}=-\lim _{T \rightarrow \infty} \frac{1}{T} \ln \left\langle W_{3 \mathrm{Q}}\left[U_{\mu}(s)\right]\right\rangle
$$

from the 3Q Wilson loop

$$
W_{3 Q}\left[U_{\mu}(s)\right] \equiv \frac{1}{3 !} \sum_{a, b, c} \sum_{a^{\prime} b^{\prime} c^{\prime}} \epsilon_{a b c} \epsilon_{a^{\prime} b^{\prime} c^{\prime}} X_{1}^{a a^{\prime}} X_{2}^{b b^{\prime}} X_{3}^{c c^{\prime}} .
$$

Here, $X_{k} \equiv \prod_{\Gamma_{k}} U_{\mu}(s)$ is the path-ordered product of the link variables along the path denoted by $\Gamma_{k}$ in Fig. 1(b). The $3 \mathrm{Q}$ Wilson loop represents that the gauge-invariant $3 \mathrm{Q}$ state is generated at $t=0$ and is annihilated at $t=T$ with the three quarks spatially fixed in $\mathbb{R}^{3}$ for $0<t<T$. We note that the potential $V_{3 \mathrm{Q}}$ is independent of the choice of the junction point $O[2,3]$, which is different from the physical junction at the Fermat point.

As shown in Fig. 1(c), we put three quarks on $(i, 0,0)$, $(0, j, 0)$, and $(0,0, k)$ in $\mathbb{R}^{3}$ with $1 \leq i \leq j \leq k \leq L / 2$ in lattice units and set the junction point $O$ at the origin $(0,0,0)$. For the calculation of the 3Q Wilson loop, we use the translational, the rotational, and the reflection symmetries on the lattices. Here, we deal with 101 and 211 different patterns of $3 \mathrm{Q}$ systems at $\beta=5.8$ and 6.0 , respectively, based on well-converged data of $\left\langle W_{3 \mathrm{Q}}\right\rangle$.

We extract $V_{3 \mathrm{Q}}$ from the least-squares fit with the singleexponential form $\left\langle W_{3 \mathrm{Q}}(T)\right\rangle=\tilde{C} e^{-V_{3 Q} T}$. Here, we choose the fit range of $T_{\min } \leq T \leq T_{\max }$ such that the stability of the so-called effective mass

$$
V_{3 \mathrm{Q}}^{\mathrm{eff}}(T) \equiv \ln \frac{\left\langle W_{3 \mathrm{Q}}(T)\right\rangle}{\left\langle W_{3 \mathrm{Q}}(T+1)\right\rangle}
$$

is observed in the range $T_{\min } \leq T \leq T_{\max }-1$. On the error estimate, we use the jackknife method.

For the accurate calculation of the $3 \mathrm{Q}$ potential with finite $T$, we apply here the gauge-invariant smearing method [2,3,5,34], which enhances the ground-state component in the 3Q state in $W_{3 \mathrm{Q}}$. The smearing is performed as the iterative replacement of the spatial link variables $U_{i}(s) \quad(i \in 1,2,3)$ by the obscured link variables $\bar{U}_{i}(s) \in \mathrm{SU}(3)$, which maximizes $\operatorname{Re} \operatorname{tr}\left[\bar{U}_{i}^{\dagger}(s) V_{i}(s)\right]$ with

$$
V_{i}(s) \equiv \alpha U_{i}(s)+\sum_{j \neq i} \sum_{ \pm} U_{ \pm j}(s) U_{i}(s \pm \hat{j}) U_{ \pm j}^{\dagger}(s \pm \hat{i}) .
$$

Here, we denote $U_{-j}(s) \equiv U_{j}^{\dagger}(s-\hat{j})$. (For the details of the smearing method, see Secs. III B and IIIC in Ref. [3].)

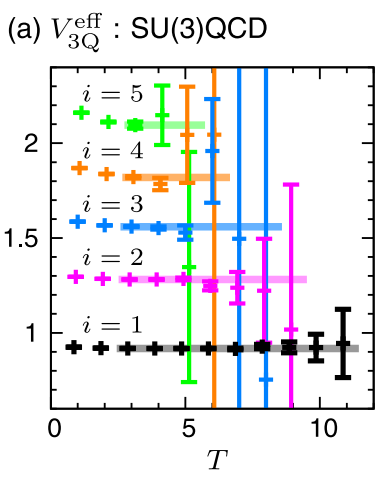

(b) $V_{3 Q}^{\text {eff }}:$ Abelian part

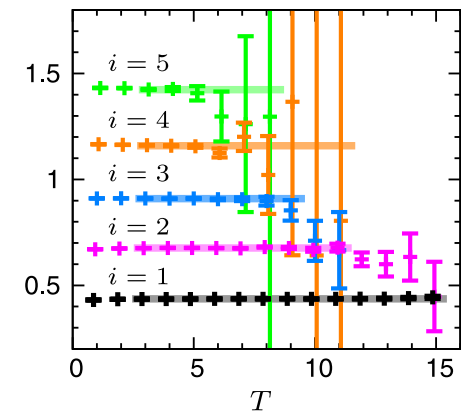

FIG. 4 (color online). Typical examples of effective mass plots of the $3 \mathrm{Q}$ potential for (a) SU(3) QCD and (b) the Abelian part in lattice units. Here, three quarks are put on the equilateral-triangle configuration, i.e., $i=j=k=1, \ldots, 5$ in Fig. 1(c), on a $16^{3} 32$ lattice at $\beta=5.8$. The solid horizontal lines denote the obtained values of $V_{3 \mathrm{Q}}$ and $V_{3 \mathrm{Q}}^{\mathrm{Abel}}$ and are extended in the corresponding fit range of $T_{\min } \leq T \leq T_{\max }-1$.

For the case of $\beta=5.8$ on $16^{3} 32$, we adopt the smearing parameter $\alpha=2.3$ and choose the iteration number $N_{\text {smr }}=$ 25 and 4 for SU(3) QCD and the Abelian part, respectively, so as to largely enhance the ground-state overlap for each part. We have confirmed that the results are almost unchanged by changing the iteration number $N_{\mathrm{smr}}$.

Similarly, we also calculate the MA projection of the $3 \mathrm{Q}$ potential

$$
V_{3 \mathrm{Q}}^{\mathrm{Abel}}=-\lim _{T \rightarrow \infty} \frac{1}{T} \ln \left\langle W_{3 \mathrm{Q}}\left[u_{\mu}(s)\right]\right\rangle
$$

from the Abelian 3Q Wilson loop in the MA gauge, $W_{3 Q}\left[u_{\mu}(s)\right]$, which is invariant under the residual Abelian gauge transformation (5). By way of illustration, we show in Fig. 4 the effective mass plot for each part at $\beta=5.8$ on $16^{3} 32$.

\section{ABELIAN DOMINANCE OF QUARK CONFINEMENT IN 3Q POTENTIAL}

In this section, we show the numerical results of QQ $\bar{Q}$ and 3Q systems in $\mathrm{SU}(3)$ quenched lattice QCD at $\beta=5.8$ on $16^{3} 32$. Figure 5(a) shows the QQ potential $V(r)$ and the Abelian part $V^{\text {Abel }}(r)$. All the lattice data of $V(r)$ are well reproduced by the Coulomb-plus-linear ansatz (1) with the best-fit parameter set listed in Table II. For a larger interquark distance $r$ than $1 \mathrm{fm}, V(r)$ is simply described by the linear quark-confining potential $\sigma r+C$ [upper straight line in Fig. 5(a)]. Figure 5(a) illuminates "perfect Abelian dominance" of confinement in the QQ potential, which was reported in Ref. [28], because the Abelian part $V^{\text {Abel }}(r)$ has a significant agreement with $\sigma r+C^{\prime}$ [lower straight line in Fig. 5(a)] at large distances.

We note that the Abelian dominance of the QQ'-confining force does not necessarily mean that of the $3 \mathrm{Q}$-confining force because one cannot superpose solutions in QCD even 
THREE-QUARK POTENTIAL AND ABELIAN DOMINANCE ...

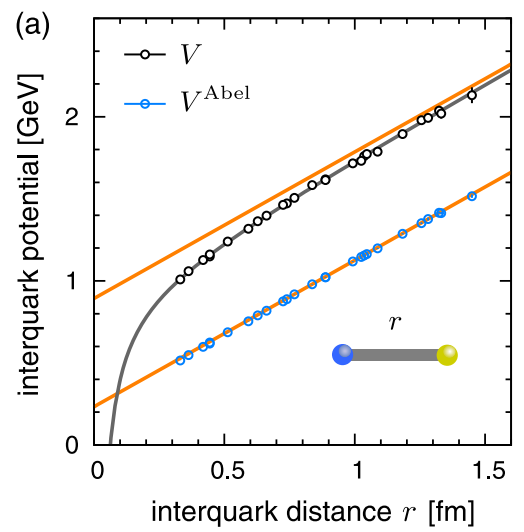

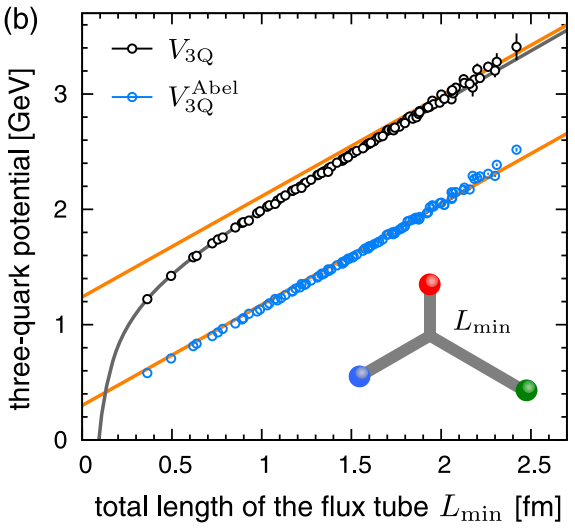

PHYSICAL REVIEW D 92, 034511 (2015)

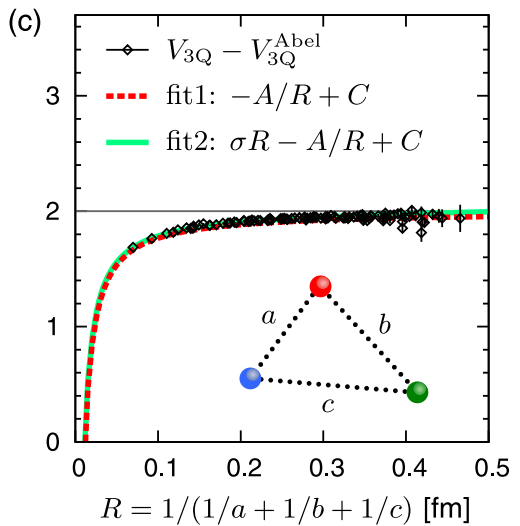

FIG. 5 (color online). MA projection of (a) QQ and (b) 3Q potentials in SU(3) quenched lattice QCD at $\beta=5.8$ on $16^{3} 32$. In each panel, the black and blue circles denote the original SU(3) potential and the Abelian part, respectively. The gray curves are obtained by the best fit with Eqs. (1) and (11) (Y ansatz), as listed in Table II. The slopes of the parallel orange lines for the panels (a) and (b) are $\sigma$ and $\sigma_{3 \mathrm{Q}}$, respectively. (c) Fit analysis of $V_{3 \mathrm{Q}}-V_{3 \mathrm{Q}}^{\text {Abel }}$ (black diamonds) to illustrate the equivalence $\sigma_{3 \mathrm{Q}} \simeq \sigma_{3 \mathrm{Q}}^{\text {Abel }}$ between the three-body string tension and its Abelian part in the baryonic 3Q potential. The red dashed and the green solid curves are the best fits with the pure Coulomb ansatz (13) and the Coulomb-plus-linear ansatz, respectively.

at the classical level. Indeed, a 3Q system cannot be described by the superposition of the interaction between two quarks, as is suggested from the functional form (2) of the $3 \mathrm{Q}$ potential $[2,3]$. We find, however, Abelian dominance of the 3Q-confining force with an accuracy within a few percent deviation as described below.

Figure 5(b) shows the 3Q potential $V_{3 \mathrm{Q}}$ and the Abelian part $V_{3 \mathrm{Q}}^{\text {Abel }}$ plotted against the total length of the flux tube, $L_{\min }$. All the lattice data of $V_{3 \mathrm{Q}}$ are approximately described by a single-valued function of $L_{\min }$, although $V_{3 \mathrm{Q}}$ generally depends on the relative position of the three quarks. The main reason is that the three-body confinement term $\sigma_{3 \mathrm{Q}} L_{\min }$ is relevant in the $\mathrm{Y}$ ansatz (2) except for short distances. When the $3 \mathrm{Q}$ system forms an equilateral triangle, one finds $L_{\min }=\sqrt{3}\left|\mathbf{r}_{i}-\mathbf{r}_{j}\right|$ for any $i \neq j$, and the Y ansatz (2) becomes

$$
V_{3 \mathrm{Q}}\left(\mathbf{r}_{1}, \mathbf{r}_{2}, \mathbf{r}_{3}\right)=\sigma_{3 \mathrm{Q}} L_{\min }-3 \sqrt{3} \frac{A_{3 \mathrm{Q}}}{L_{\min }}+C_{3 \mathrm{Q}}
$$

Since $V_{3 Q}$ approximately obeys a single-valued function of $L_{\min }$, all the lattice data are well reproduced by Eq. (11) with the best-fit parameter set as listed in Table II, other than the equilateral-triangle 3Q systems. When the total flux-tube length $L_{\min }$ is larger than $1 \mathrm{fm}$, $V_{3 \mathrm{Q}}$ is described by the linear $3 \mathrm{Q}$-confining potential $\sigma_{3 \mathrm{Q}} L_{\min }+C_{3 \mathrm{Q}} \quad$ [upper straight line in Fig. 5(b)]. Remarkably, the Abelian part $V^{\text {Abel }}(r)$ has a significant agreement with $\sigma_{3 \mathrm{Q}} L_{\min }+C_{3 \mathrm{Q}}^{\prime}$ [lower straight line in Fig. 5(b)] at large distances, which is plausible evidence for $\sigma_{3 \mathrm{Q}}^{\mathrm{Abel}} \simeq \sigma_{3 \mathrm{Q}}$ in the baryonic $3 \mathrm{Q}$ potential.

To demonstrate $\sigma_{3 \mathrm{Q}}^{\mathrm{Abel}} \simeq \sigma_{3 \mathrm{Q}}$ conclusively, we investigate the difference between $V_{3 \mathrm{Q}}$ and $V_{3 \mathrm{Q}}^{\mathrm{Abel}}$ at long distances as shown in Fig. 5(c). As is the case in $V_{3 \mathrm{Q}}$, the Abelian part of the $3 \mathrm{Q}$ potential has the functional form

$$
V_{3 \mathrm{Q}}^{\mathrm{Abel}}=\sigma_{3 \mathrm{Q}}^{\mathrm{Abel}} L_{\min }-\frac{A_{3 \mathrm{Q}}^{\mathrm{Abel}}}{R}+C_{3 \mathrm{Q}}^{\mathrm{Abel}},
$$

TABLE II. Fit analysis of interquark potentials in lattice units at $\beta=5.8$ (i.e., $a \simeq 0.15 \mathrm{fm}$ ) on $16^{3} 32$ and $\beta=6.0$ (i.e., $a \simeq 0.10 \mathrm{fm}$ ) on $20^{3} 32$. The best-fit parameter sets $(\sigma, A, C)$ of the QQ potential $V$ and the Abelian part $V^{\text {Abel }}$ are listed with the functional form (1). The best-fit parameter sets $\left(\sigma_{3 \mathrm{Q}}, A_{3 \mathrm{Q}}, C_{3 \mathrm{Q}}\right)$ of the $3 \mathrm{Q}$ potential $V_{3 \mathrm{Q}}$ and the Abelian part $V_{3 \mathrm{Q}}^{\mathrm{Abel}}$ are listed with the $\mathrm{Y}$ ansatz (2). The label of (equi. triangle) means the fit analysis only with the lattice data of equilateral-triangle $3 \mathrm{Q}$ configurations. $N_{Q}$ is the number of different

\begin{tabular}{|c|c|c|c|c|c|c|c|c|c|}
\hline \multirow[b]{2}{*}{$\beta$} & & \multirow[b]{2}{*}{$N_{\mathrm{Q}}$} & \multicolumn{3}{|c|}{$\mathrm{SU}(3)$} & \multicolumn{3}{|c|}{ Abelian part } & \multirow[b]{2}{*}{$\sigma^{\mathrm{Abel}} / \sigma$} \\
\hline & & & $\sigma$ & $A$ & C & $\sigma^{\text {Abel }}$ & $A^{\text {Abel }}$ & $C^{\text {Abel }}$ & \\
\hline \multirow[t]{3}{*}{$\overline{5.8}$} & QQ̄ & 26 & $0.099(2)$ & $0.30(3)$ & $0.67(2)$ & $0.098(1)$ & $0.043(12)$ & $0.187(7)$ & $0.99(3)$ \\
\hline & $3 \mathrm{Q}$ (equi. triangle) & 5 & $0.097(1)$ & $0.118(3)$ & $0.93(1)$ & $0.098(3)$ & $-0.001(8)$ & $0.19(2)$ & $1.01(3)$ \\
\hline & $3 \mathrm{Q}$ & 101 & 0.0997(4) & $0.109(1)$ & $0.905(4)$ & $0.0967(5)$ & $0.006(2)$ & $0.213(5)$ & $0.97(1)$ \\
\hline \multirow[t]{3}{*}{6.0} & QQ̄ & 39 & $0.0472(6)$ & $0.289(10)$ & $0.658(5)$ & $0.0457(2)$ & $0.050(3)$ & $0.183(2)$ & $0.97(1)$ \\
\hline & $3 \mathrm{Q}$ (equi. triangle) & 8 & $0.0471(10)$ & $0.121(3)$ & $0.936(9)$ & $0.0455(12)$ & $0.014(4)$ & $0.233(12)$ & $0.97(3)$ \\
\hline & $3 Q$ & 211 & $0.0480(3)$ & $0.113(1)$ & $0.917(3)$ & $0.0456(2)$ & $0.013(1)$ & $0.232(2)$ & $0.95(1)$ \\
\hline
\end{tabular}
patterns of QQ or $3 \mathrm{Q}$ systems. The string tension ratio $\sigma^{\mathrm{Abel}} / \sigma$ is listed in the last column. 
NAOYUKI SAKUMICHI AND HIDEO SUGANUMA

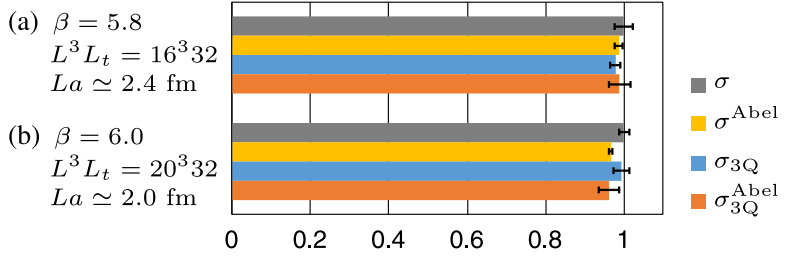

FIG. 6 (color online). Comparison of string tensions of the QQ and $3 \mathrm{Q}$ potentials for $\mathrm{SU}(3) \mathrm{QCD}$ and the Abelian part. Here, $\sigma$ and $\sigma_{\mathrm{Abel}}$ are the string tensions of the QQ potential for SU(3) QCD and the Abelian part, respectively. Similarly, $\sigma_{3 \mathrm{Q}}$ and $\sigma_{3 \mathrm{Q}}$ are the string tensions of the 3Q potential for SU(3) QCD and the Abelian part, respectively. In both cases of (a) $\beta=5.8$ on the $16^{3} 32$ lattice and (b) $\beta=6.0$ on the $16^{3} 32$ lattice, we find that the string tensions are equivalent within a few percent deviation: $\sigma \simeq \sigma^{\mathrm{Abel}} \simeq \sigma_{3 \mathrm{Q}} \simeq \sigma_{3 \mathrm{Q}}^{\mathrm{Abel}}$.

where $1 / R \equiv \sum_{i<j} 1 /\left|\mathbf{r}_{i}-\mathbf{r}_{j}\right|$ [35]. If the Abelian dominance of the $3 \mathrm{Q}$ potential is exact, i.e., $\sigma_{3 \mathrm{Q}}^{\mathrm{Abel}}=\sigma_{3 \mathrm{Q}}$, one has to observe

$$
\Delta V_{3 \mathrm{Q}} \equiv V_{3 \mathrm{Q}}-V_{3 \mathrm{Q}}^{\mathrm{Abel}}=-\frac{\Delta A_{3 \mathrm{Q}}}{R}+\Delta C_{3 \mathrm{Q}}
$$

where $\Delta A_{3 \mathrm{Q}} \equiv A_{3 \mathrm{Q}}-A_{3 \mathrm{Q}}^{\mathrm{Abel}}$ and $\Delta C_{3 \mathrm{Q}} \equiv C_{3 \mathrm{Q}}-C_{3 \mathrm{Q}}^{\mathrm{Abel}}$. Then, we try a fit analysis to $\Delta V_{3 \mathrm{Q}}$ with the pure Coulomb ansatz (13) (fit 1) and the Coulomb-plus-linear ansatz, $\Delta \sigma_{3 \mathrm{Q}}^{\prime} R-\Delta A_{3 \mathrm{Q}}^{\prime} / R+\Delta C_{3 \mathrm{Q}}^{\prime}$ (fit 2), in Fig. 5(c). Fits 1 and 2 reveal that $\Delta V_{3 \mathrm{Q}}$ has almost zero string tension, $\Delta \sigma_{3 \mathrm{Q}}^{\prime} \simeq 0$, and is well reproduced by the pure Coulomb ansatz (13). Therefore, we conclude that there is no difference between the string tensions in $V_{3 Q}$ and $V_{3 Q}^{\text {Abel }}$, i.e., $\sigma_{3 \mathrm{Q}}^{\text {Abel }} \simeq \sigma_{3 \mathrm{Q}}$, with an accuracy within a few percent deviation. [We remark that Fig. 5(c) would be plausible evidence for the exact equivalence of $\sigma_{3 \mathrm{Q}}^{\mathrm{Abel}}=\sigma_{3 \mathrm{Q}}$.]

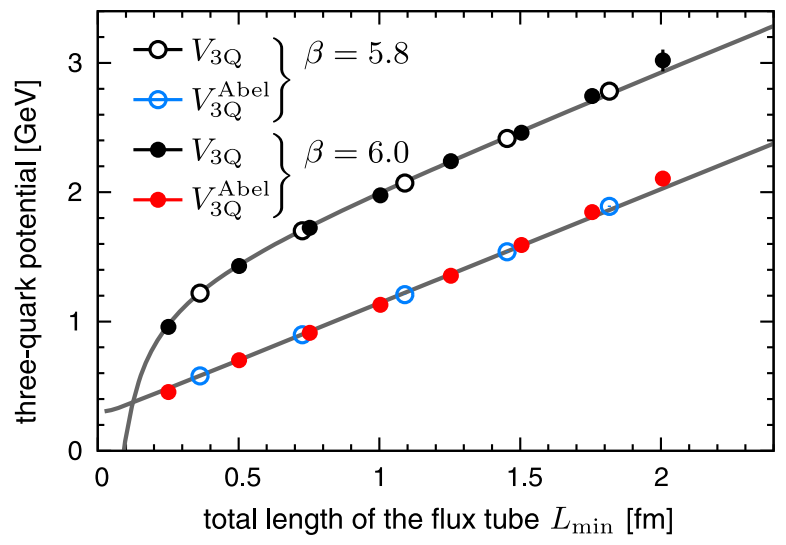

FIG. 7 (color online). The MA projection of the 3Q potential for equilateral-triangular configurations plotted against $L_{\min }$ for $\beta=5.8$ and 6.0 in the physical unit. The curves are obtained by the best fit with Eq. (11) for the $\beta=5.8$ data, as listed in Table II.
PHYSICAL REVIEW D 92, 034511 (2015)

TABLE III. A part of the lattice data of the $3 \mathrm{Q}$ potential $V_{3 \mathrm{Q}}$ and the Abelian part $V_{3 \mathrm{Q}}^{\mathrm{Abel}}$ restricted for the equilateral-triangle configuration, i.e., $i=j=k$ in Fig. 1(c), in lattice units.

\begin{tabular}{lrlllll}
\hline \hline & & \multicolumn{2}{c}{$16^{3} 32$ at $\beta=5.8$} & & \multicolumn{2}{c}{$20^{3} 32$ at $\beta=6.0$} \\
$(i, j, k)$ & $L_{\text {min }}$ & $V_{3 \mathrm{Q}}$ & $V_{3 \mathrm{Q}}^{\text {Abel }}$ & & $V_{3 \mathrm{Q}}$ & \multicolumn{1}{c}{$V_{3 \mathrm{Q}}^{\text {Abel }}$} \\
\hline$(1,1,1)$ & 2.45 & $0.9176(2)$ & $0.4361(1)$ & & $0.7943(3)$ & $0.3140(1)$ \\
$(2,2,2)$ & 4.90 & $1.2812(9)$ & $0.6765(4)$ & & $1.0393(8)$ & $0.4425(2)$ \\
$(3,3,3)$ & 7.35 & $1.559(2)$ & $0.9095(9)$ & & $1.193(2)$ & $0.5521(3)$ \\
$(4,4,4)$ & 9.80 & $1.819(6)$ & $1.159(2)$ & & $1.323(3)$ & $0.6648(6)$ \\
$(5,5,5)$ & 12.24 & $2.10(2)$ & $1.424(4)$ & & $1.460(6)$ & $0.782(1)$ \\
$(6,6,6)$ & 14.70 & $\ldots$ & $\ldots$ & & $1.58(1)$ & $0.906(2)$ \\
$(7,7,7)$ & 17.15 & $\ldots$ & $\ldots$ & & $1.72(1)$ & $1.037(2)$ \\
$(8,8,8)$ & 19.60 & $\ldots$ & $\ldots$ & $1.87(4)$ & $1.172(3)$ \\
\hline \hline
\end{tabular}

To see the finite lattice-spacing effect, we also perform $\mathrm{SU}(3)$ quenched lattice QCD at $\beta=6.0$ on $20^{3} 32$ and summarize the results in Table II and Fig. 6(b). We find again $\sigma_{3 \mathrm{Q}}^{\mathrm{Abel}} \simeq \sigma_{3 \mathrm{Q}}$, and thus $\sigma_{3 \mathrm{Q}}^{\mathrm{Abel}} \simeq \sigma_{3 \mathrm{Q}}$ is expected to be true in the finer lattice spacings, similar to the case of the $\mathrm{QQ}$ potential [28]. Figure 6 compares the QQ $\bar{Q}$ and $3 \mathrm{Q}$ results at $\beta=5.8$ on the $16^{3} 32$ lattice and at $\beta=6.0$ on the $20^{3} 32$ lattice. In both cases, we find that the string tensions of the QQ and 3Q potentials for the SU(3) and Abelian part are equivalent within a few percent deviation: $\sigma \simeq \sigma^{\mathrm{Abel}} \simeq$ $\sigma_{3 \mathrm{Q}} \simeq \sigma_{3 \mathrm{Q}}^{\mathrm{Abel}}$. To be exact, in the case of $\beta=6.0$ on the $20^{3} 32$ lattice, the string tensions of the Abelian part $\left(\sigma^{\mathrm{Abel}} \simeq \sigma_{3 \mathrm{Q}}^{\mathrm{Abel}}\right)$ are about $3 \%$ smaller than $\mathrm{SU}(3) \mathrm{QCD}$ $\left(\sigma \simeq \sigma_{3 \mathrm{Q}}\right)$ because the physical spatial size is slightly small $(L a \simeq 2.0 \mathrm{fm}$ ). This physical-spatial-size effect is expected from the result of the QQ potential [28] (see Fig. 3).

For a visual demonstration, we restrict ourselves on equilateral-triangular 3Q configurations and show their lattice results of $V_{3 \mathrm{Q}}$ and $V_{3 \mathrm{Q}}^{\text {Abel }}$ at $\beta=5.8$ and 6.0 in the physical unit in Fig. 7, where an irrelevant constant is shifted. For each of $V_{3 \mathrm{Q}}$ and $V_{3 \mathrm{Q}}^{\text {Abel }}$, both lattice data are found to be well reproduced by a single curve. We list their raw data in Table III and add the fit result in Table II.

To conclude, we thus find Abelian dominance of the string tension, $\sigma_{3 \mathrm{Q}}^{\mathrm{Abel}} \simeq \sigma_{3 \mathrm{Q}}$, with an accuracy within a few percent deviation in the baryonic $3 \mathrm{Q}$ potential in $\mathrm{SU}(3)$ quenched lattice QCD for $\beta=5.8$ on $16^{3} 32$ and $\beta=6.0$ on $20^{3} 32$, as shown in Table II.

\section{SUMMARY AND CONCLUDING REMARKS}

We have studied the MA projection of quark confinement in the baryonic 3Q potential in the SU(3) quenched lattice QCD with $\beta=5.8$ on $16^{3} 32$ and $\beta=6.0$ on $20^{3} 32$ for more than 300 different $3 \mathrm{Q}$ systems in total, with 1000-2000 gauge configurations. (Note also that the lattice data of $V_{3 \mathrm{Q}}$ themselves are fairly accurate, because of the high statistics.) Remarkably, we have found 
Abelian dominance of the string tension with an accuracy within a few percent deviation, $\sigma \simeq \sigma^{\text {Abel }} \simeq \sigma_{3 \mathrm{Q}} \simeq \sigma_{3 \mathrm{Q}}^{\text {Abel }}$, in $\mathrm{QQ}$ and 3Q potentials simultaneously on these lattices. (For a more definite conclusion, it is desired to perform similar studies with larger and finer lattices.) Thus, despite the non-Abelian nature of QCD, quark confinement is entirely kept in the Abelian sector of QCD in the MA gauge. In other words, Abelianization of QCD can be realized without the loss of the quark-confining force via the MA projection. This fact would be meaningful to understand the confinement mechanism in the non-Abelian gauge theory of QCD. Furthermore, the Abelian dominance for both QQ $\bar{Q}$ and $3 \mathrm{Q}$ potentials indicates a universality of the confinement mechanism for the wide category of hadrons in terms of Abelianization of QCD.

\section{ACKNOWLEDGMENTS}

The authors thank Hideaki Iida and Toru T. Takahashi. H. S. thanks V. G. Bornyakov for his valuable suggestions. N. S. is supported by a Grant-in-Aid for JSPS Fellows and by JSPS KAKENHI Grants No. 250588, No. 15K17725. H.S. is supported by the Grants-in-Aid for Scientific Research [Grants No. 23540306 and No. 15K05076] from Japan Society for the Promotion of Science. The lattice calculations were partially performed on NEC-SX8R at Osaka University. This work was partially supported by RIKEN iTHES Project.
[1] H. J. Rothe, Lattice Gauge Theories, 4th ed. (World Scientific, Singapore, 2012), and references therein.

[2] T. T. Takahashi, H. Matsufuru, Y. Nemoto, and H. Suganuma, Phys. Rev. Lett. 86, 18 (2001); T. T. Takahashi and H. Suganuma, Phys. Rev. Lett. 90, 182001 (2003); Phys. Rev. D 70, 074506 (2004).

[3] T. T. Takahashi, H. Suganuma, Y. Nemoto, and H. Matsufuru, Phys. Rev. D 65, 114509 (2002).

[4] J. M. Cornwall, Phys. Rev. D 69, 065013 (2004).

[5] G. S. Bali and K. Schilling, Phys. Rev. D 47, 661 (1993).

[6] For a review article, see G. S. Bali, Phys. Rep. 343, 1 (2001).

[7] F. Okiharu, H. Suganuma, and T. T. Takahashi, Phys. Rev. D 72, 014505 (2005).

[8] A. Casher, H. Neuberger, and S. Nussinov, Phys. Rev. D 20, 179 (1979).

[9] H. Ichie, V. Bornyakov, T. Streuer, and G. Schierholz, Nucl. Phys. A721, C899 (2003).

[10] V. G. Bornyakov, H. Ichie, Y. Mori, D. Pleiter, M. I. Polikarpov, G. Schierholz, T. Streuer, H. Stüben, and T. Suzuki (DIK Collaboration), Phys. Rev. D 70, 054506 (2004).

[11] P. O. Bowman and A. P. Szczepaniak, Phys. Rev. D 70, 016002 (2004).

[12] M. Cardoso, N. Cardoso, and P. Bicudo, Phys. Rev. D 86, 014503 (2012); N. Cardoso, M. Cardoso, and P. Bicudo, Phys. Rev. D 88, 054504 (2013).

[13] M. S. Cardaci, P. Cea, L. Cosmai, R. Falcone, and A. Papa, Phys. Rev. D 83, 014502 (2011).

[14] O. Andreev, Phys. Rev. D 78, 065007 (2008).

[15] Y. Nambu, Phys. Rev. D 10, 4262 (1974); G. 't Hooft, High Energy Physics (Editorice Compositori, Bologna, Italy, 1975); S. Mandelstam, Phys. Rep. 23, 245 (1976).

[16] N. Seiberg and E. Witten, Nucl. Phys. B426, 19 (1994); B431, 484 (1994).
[17] G. 't Hooft, Nucl. Phys. B190, 455 (1981).

[18] Z. F. Ezawa and A. Iwazaki, Phys. Rev. D 25, 2681 (1982).

[19] A. S. Kronfeld, G. Schierholz, and U.-J. Wiese, Nucl. Phys. B293, 461 (1987); A. S. Kronfeld, M. L. Laursen, G. Schierholz, and U.-J. Wiese, Phys. Lett. B 198, 516 (1987).

[20] T. Suzuki and I. Yotsuyanagi, Phys. Rev. D 42, 4257 (1990).

[21] J. D. Stack, S. D. Neiman, and R. J. Wensley, Phys. Rev. D 50, 3399 (1994).

[22] K. Amemiya and H. Suganuma, Phys. Rev. D 60, 114509 (1999).

[23] O. Miyamura, Phys. Lett. B 353, 91 (1995).

[24] R. M. Woloshyn, Phys. Rev. D 51, 6411 (1995).

[25] H. Suganuma, A. Tanaka, S. Sasaki, and O. Miyamura, Nucl. Phys. B, Proc. Suppl. 47, 302 (1996).

[26] J. D. Stack, W. W. Tucker, and R. J. Wensley, Nucl. Phys. B639, 203 (2002).

[27] V. G. Bornyakov et al. (DIK Collaboration), Phys. Rev. D 70, 074511 (2004).

[28] N. Sakumichi and H. Suganuma, Phys. Rev. D 90, 111501 (2014).

[29] S. Kato, K.-I. Kondo, and A. Shibata, Phys. Rev. D 91, 034506 (2015).

[30] F. Brandstater, G. Schierholz, and U.-J. Wiese, Phys. Lett. B 272, 319 (1991).

[31] C. Bonati and M. D'Elia, Nucl. Phys. B877, 233 (2013).

[32] H. Ichie and H. Suganuma, Nucl. Phys. B548, 365 (1999); Phys. Rev. D 60, 077501 (1999).

[33] J. Vijande, A. Valcarce, and H. Garcilazo, Phys. Rev. D 90, 094004 (2014).

[34] M. Albanese et al. (APE Collaboration), Phys. Lett. B 192, 163 (1987).

[35] N. Brambilla, J. Ghiglieri, and A. Vairo, Phys. Rev. D 81, 054031 (2010); N. Brambilla, F. Karbstein, and A. Vairo, Phys. Rev. D 87, 074014 (2013). 